\title{
Unveiling Jet Topology via Multi-Particle Correlations
}

\author{
N. N. Ajitanand* \\ Department of Chemistry, Stony Brook University,Stony Brook, NY 11794,USA \\ E-mail: ajit@mail.chem. sunysb.edu
}

\begin{abstract}
There is now a considerable body of evidence to indicate that in heavy ion collisions at RHIC energies a strongly interacting state of matter resembling a near-perfect liquid (termed sQGP) is formed. Along with the soft collisions responsible for the creation of this medium, there are hard parton-parton collisions resulting in jets which provide a natural probe of the medium. Considerable effort has gone into a detailed study of the properties of these jets and their medium induced modification. In particular, the study of jet topolgy has drawn considerable attention since there are several theoretical predictions of the effect of the medium on jet shape. Multi-particle correlations are a potential tool for conducting such investigations. In this work, the results of the study of two and three particle correlations applied to RHIC data will be discussed. Such studies are likely to be extended to the high jet multiplicity environment at the LHC where the properties of the medium could well be significantly different from the sQGP found at RHIC.
\end{abstract}

High-pT physics at LHC

March 23-27 2007

University of Jyväskylä, Jyväskylä, Finland

${ }^{*}$ Speaker. 


\section{Introduction}

There is a general consensus that in $\mathrm{Au}+\mathrm{Au}$ collisions at RHIC, a state of matter is created which can be characterized as a strongly interacting, low viscosity fluid of quarks and gluons (termed sQGP) [1]. A topic of current interest is the medium response to hard parton-parton collisions. It has been theorized that such a response may include the creation of conical flow (a "sonic boom")[2] in the medium. An alternate scenario is the possibility of a deflection of the away side jet induced by interactions with the flowing medium[3]. Jets have conventionally been studied via azimuthal two-particle correlations and one of the important findings of this method has been the away side modification of the di-jet shape[4]. In the following, we describe the effectiveness of three-particle correlations to diffrentiate between the model scenarios. We then show results from the application of the method to the analysis of RHIC data for $200 \mathrm{GeV} \mathrm{Au+Au}$ collisions.

\section{Two-particle correlations:}

A 2-particle correlation function, $C_{2}$, is a ratio of the normalized real distribution made from pairs chosen in a single event to the normalized mixed distribution where each member of the pair is chosen from a different event :

$$
C_{2}=N_{\text {real }} / N_{\text {mixed }}
$$

The $C_{2}$ for azimuthal angle correlations uses the pair variable

$$
\Delta \phi=|\phi 1-\phi 2|
$$

Under the two source model $C_{2}$ will have two components, one from flow and another from jets and can be written as

$$
C_{2}(\Delta \phi)=a_{0}[H(\Delta \phi)+J(\Delta \phi)]
$$

where $H(\Delta \phi)$ is the harmonic function representing flow. For pure elliptic flow $H(\Delta \phi)$ is given by

$$
H(\Delta \phi)=1+2 v_{2}^{(1)} v_{2}^{(2)} 2 \cos (2 \Delta \phi)
$$

where $v_{2}^{(i)}$ is the strength of the elliptic flow for particle "i".

Given the value of $v_{2}$, the value of $a_{0}$ can be determined by using the ZYAM condition[5] i.e. the jet function $J(\Delta \phi)$ is zero at its minimum. Fig. 1 and Fig. 2 demonstrate the effectiveness of using the ZYAM approach in recovering input jet shapes for normal and abnormal cases. In Fig. 2 the ability to faithfully recover jet shapes at different orientations with respect to the reaction plane is also shown.

Fig. 3 shows the result of applying this method to the de-convolution of di-hadron azimuthal correlations for $200 \mathrm{GeV} \mathrm{Au+Au} \mathrm{collisions.}$

The $p_{T}$ ranges of the particle pair are 2.5-4.0 GeV/c (trigger) and 1.0-2.5 GeV/c (associated). Note the strong away side jet modification seen for non-peripheral centralities. Fig. 4 shows the results when the low $p_{T}$ hadron is a either a meson or a baryon. The away side shapes are very similar for the two cases showing the modification is not flavor dependent.

This is consistent with the picture of medium modification effects occurring at the partonic stage of the collision. 


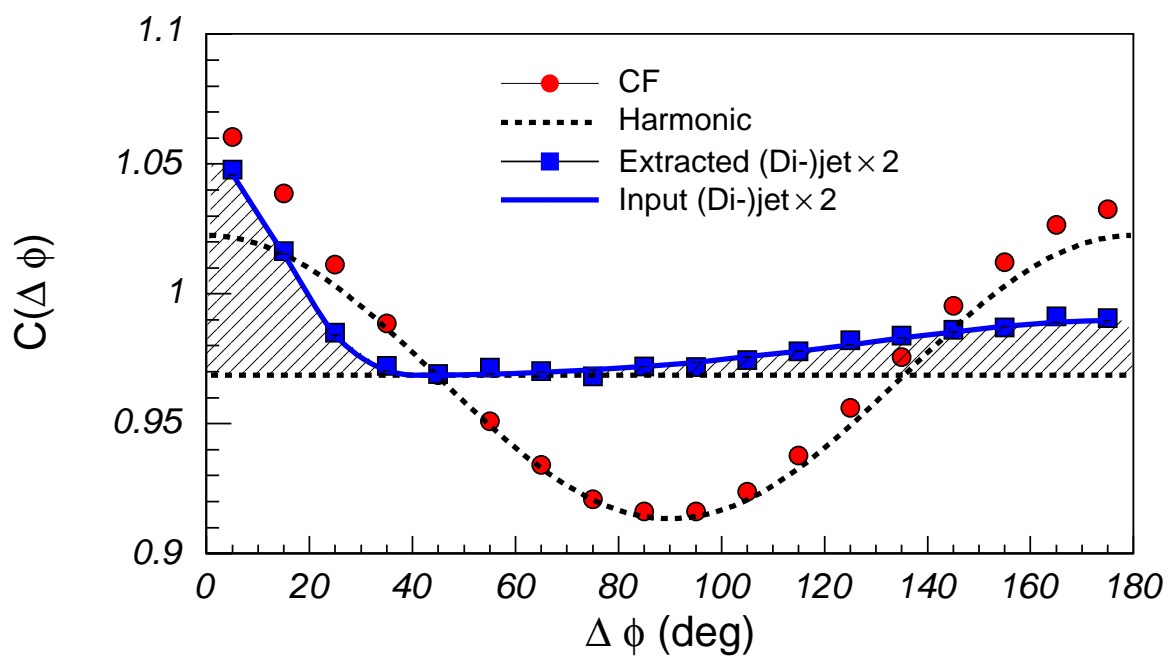

Figure 1: Demonstration of the effectiveness of the ZYAM procedure in recovering input jet correlation with a normal shape. Solid red points are for the total correlation function, dotted line is for the harmonic contribution, blue line is for the input jet function (shaded part) and the solid squares are for the recovered jet function.

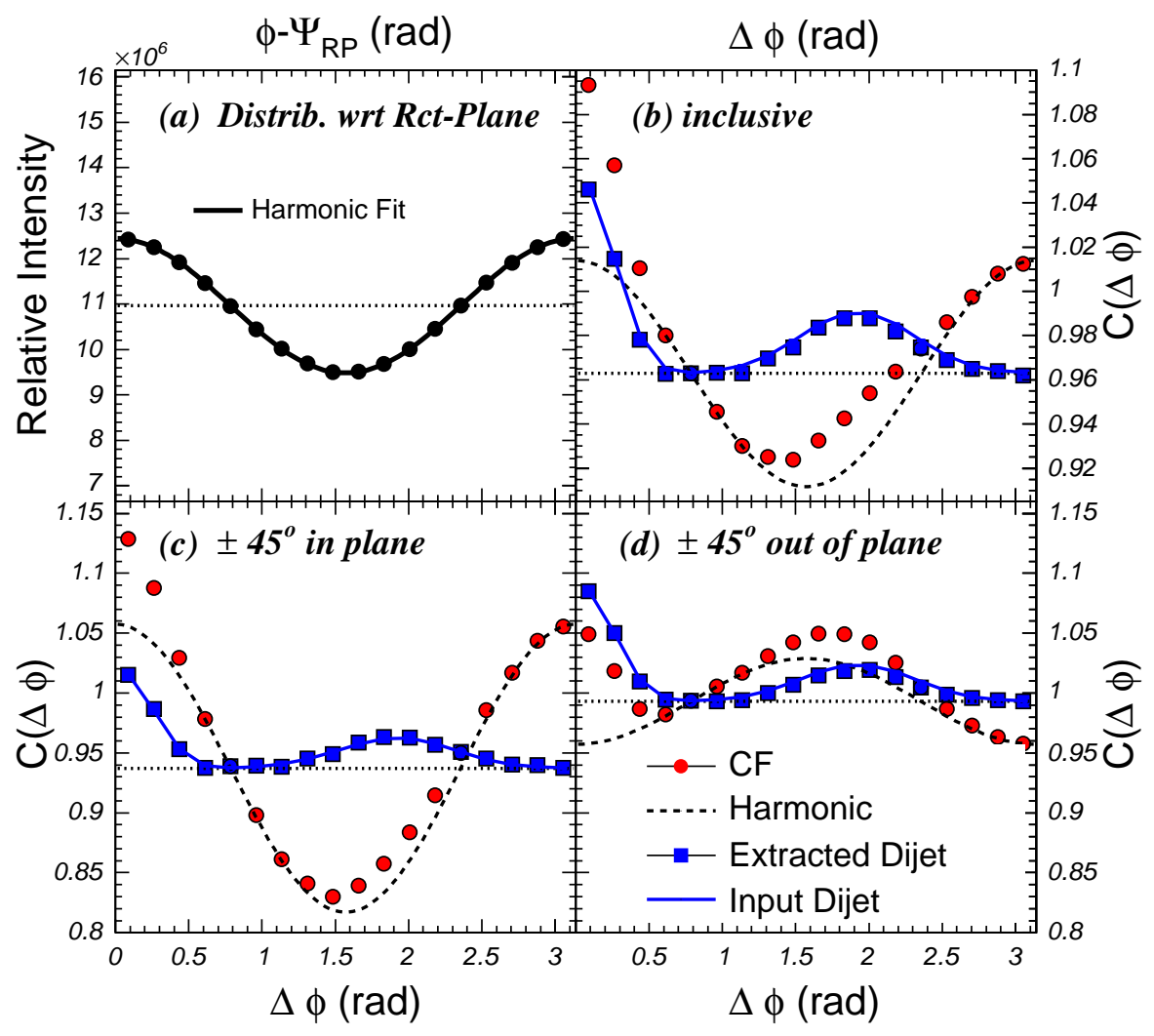

Figure 2: Demonstration of the effectiveness of the ZYAM procedure in recovering input jet correlation with an abnormal shape. Blue line is the input jet function and solid blue points are the recovered jet function. (a) shows the inclusive harmonic contribution, (b) shows jet recovery for inclusive, (c) shows jet recovery in plane and (d) shows jet recovery out of plane. 


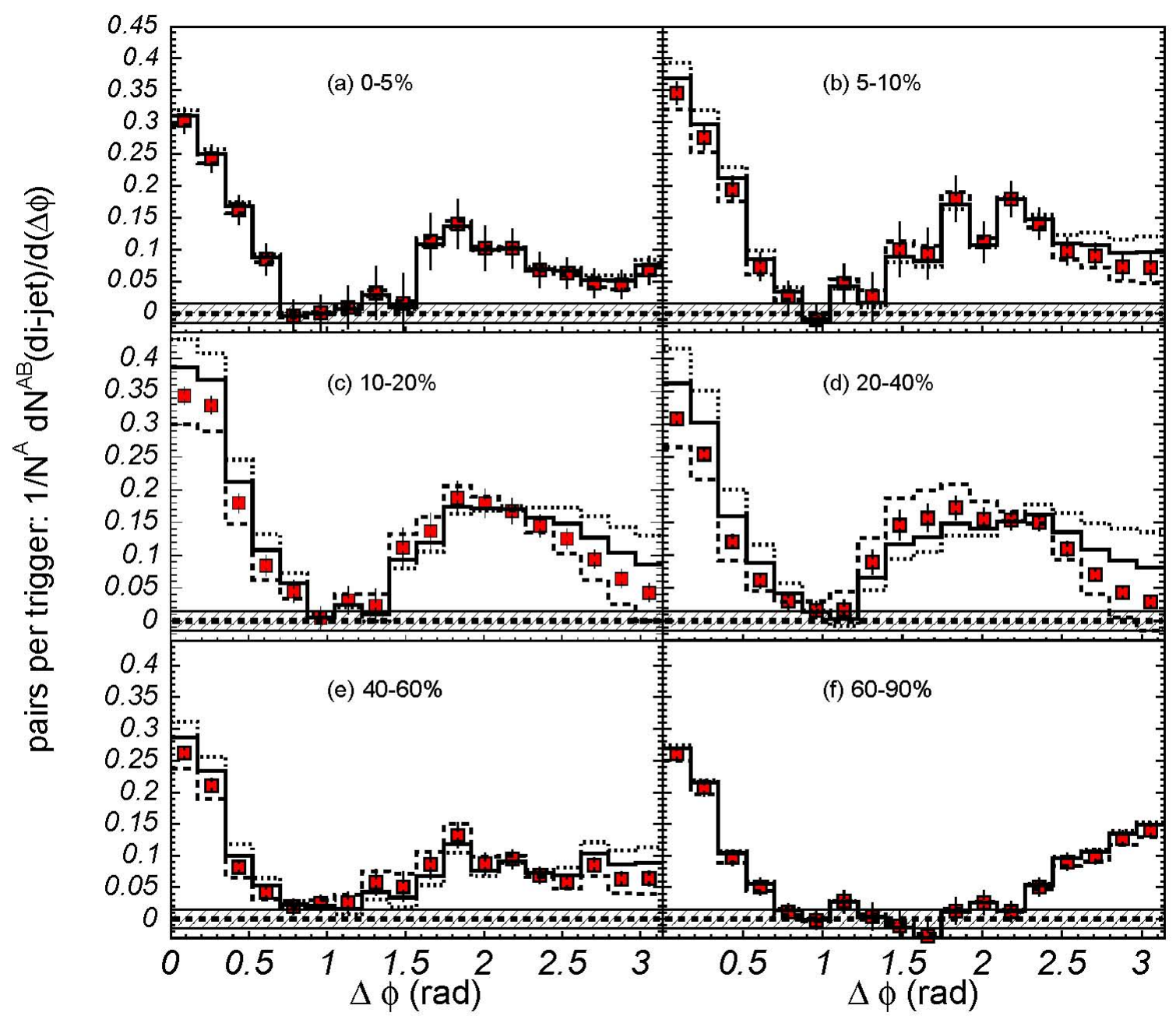

Figure 3: Jet correlation functions for $\left(2.5<p_{T}<4.0\right)$ (trigger) and $\left(1.0<p_{T}<2.5\right)$ (associated) hadron pairs in $\operatorname{sqrt}\left(s_{N N}\right)=200 \mathrm{GeV} \mathrm{Au}+\mathrm{Au}$ collisions at different centralities.

\section{Three-particle correlations:}

A 3-particle correlation function, $C_{3}$, is a ratio of the normalized real distribution made from triplets chosen in a single event to the normalized mixed distribution where each member of the triplet is chosen from a different event:

$$
C_{3}\left(\Delta \phi^{*}, \theta^{*}\right)=N_{\text {real }}\left(\Delta \phi^{*}, \theta^{*}\right) / N_{\text {mixed }}\left(\Delta \phi^{*}, \theta^{*}\right)
$$

To study jet properties we use a 3-particle correlation function built by combining a hadron in a specified high transverse momentum range $\left(2.5<p_{T}<4.0 \mathrm{GeV} / \mathrm{c}\right)$ with two associated hadrons in an adjacent low transverse momentum range $\left(1.0<p_{T}<2.5 \mathrm{GeV} / \mathrm{c}\right)$. 


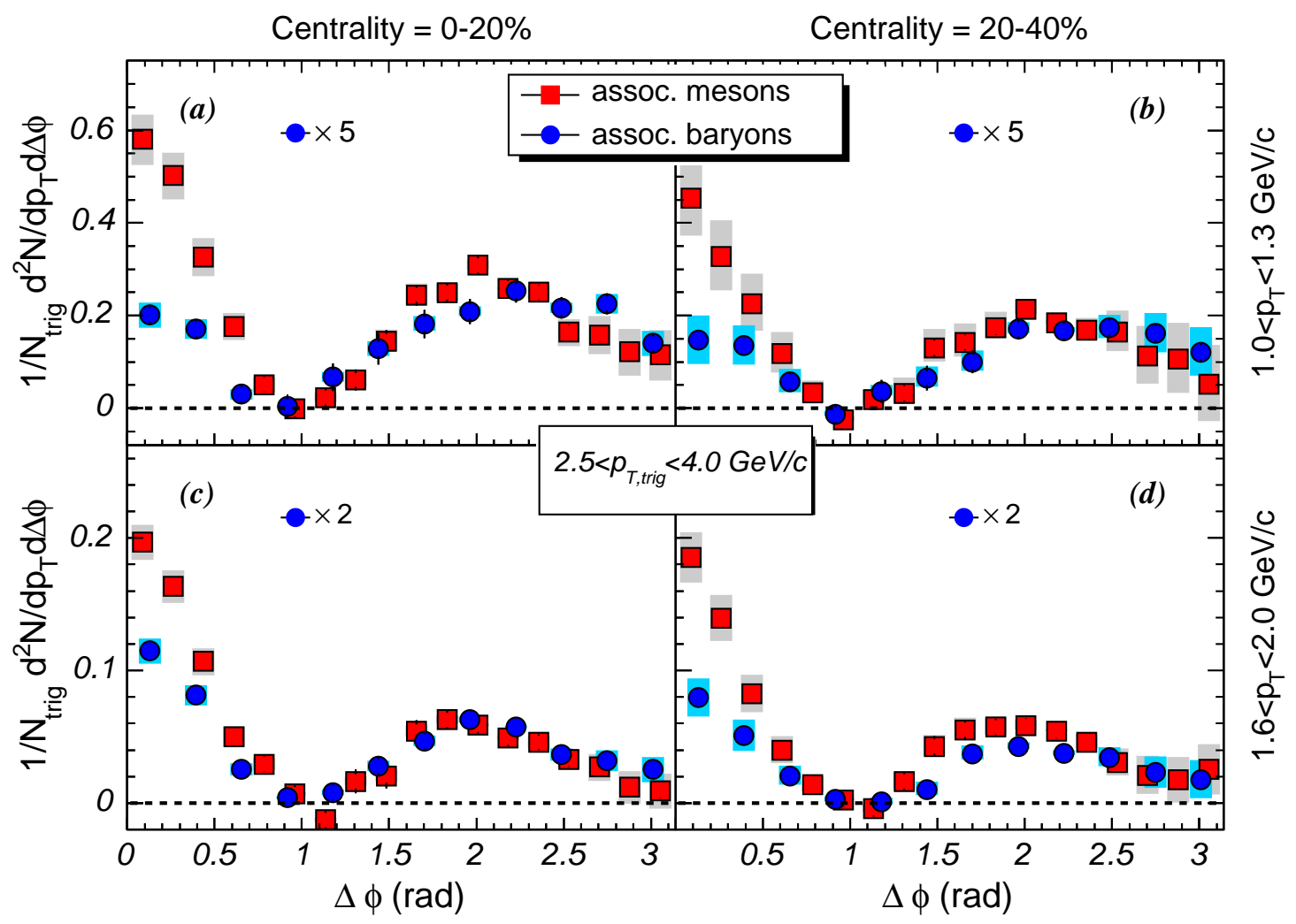

Figure 4: Jet correlation functions in $\operatorname{sqrt}\left(s_{N N}\right)=200 \mathrm{GeV} \mathrm{Au+Au} \mathrm{collisions} \mathrm{for} \mathrm{trigger} \mathrm{hadron}\left(2.5<p_{T}<\right.$ $4.0)$ with associated partner mesons (red symbols) and baryons (blue symbols) for centralities $(0-20 \%)$ and $(20-40 \%)$ in pT ranges $\left(1.0<p_{T}<1.3\right)(\mathrm{a}, \mathrm{b})$ and $\left(1.6<p_{T}<2.0\right)(\mathrm{c}, \mathrm{d})$

By transforming to the frame of reference in which the z-axis is defined by the high $p_{T}$ particle momentum vector, a two dimensional correlation function is obtained in $\theta^{*}, \Delta \phi^{*}$ space where $\theta^{*}$ is the polar angle of one of the low $p_{T}$ particles and $\Delta \phi^{*}$ is the difference of azimuthal angles of the two low $p_{T}$ particles (Fig.5). In the present approach no cuts are made on the relative angles of the associated particles. The 3-particle correlation functions can then be shown as polar plots in which $\theta^{*}$ is plotted along the radial axis and $\Delta \phi^{*}$ is plotted along the azimuthal axis.

Simulated 3-particle correlation surfaces are shown in Fig. 6 for two distinct away-side jet scenarios; (i) a "deflected jet" in which the away-side jet axis is misaligned by $60^{\circ}$ (Fig. 6a), and (ii) a "Mach cone jet" in which the leading and away-side jet axes are aligned but fragmentation is confined to a very thin hollow cone with a half angle of $60^{\circ}$ (Fig. 6c). In both scenarios the di-jet axes are back-to-back in eta. Although the standard two-particle correlations for the two cases are very similar (Fig. 6b), the 3-particle correlations show rather clear distinguishing features. The deflection model shows a peaking of the correlation strength in the $\Delta \phi^{*}=0$ region which is not the case for the cone model.

Three particle correlations, $C 3$, constructed in the data have both jet correlations as well as harmonic (flow) correlations. The harmonic correlation is removed from $C 3$ following the ZYAM 


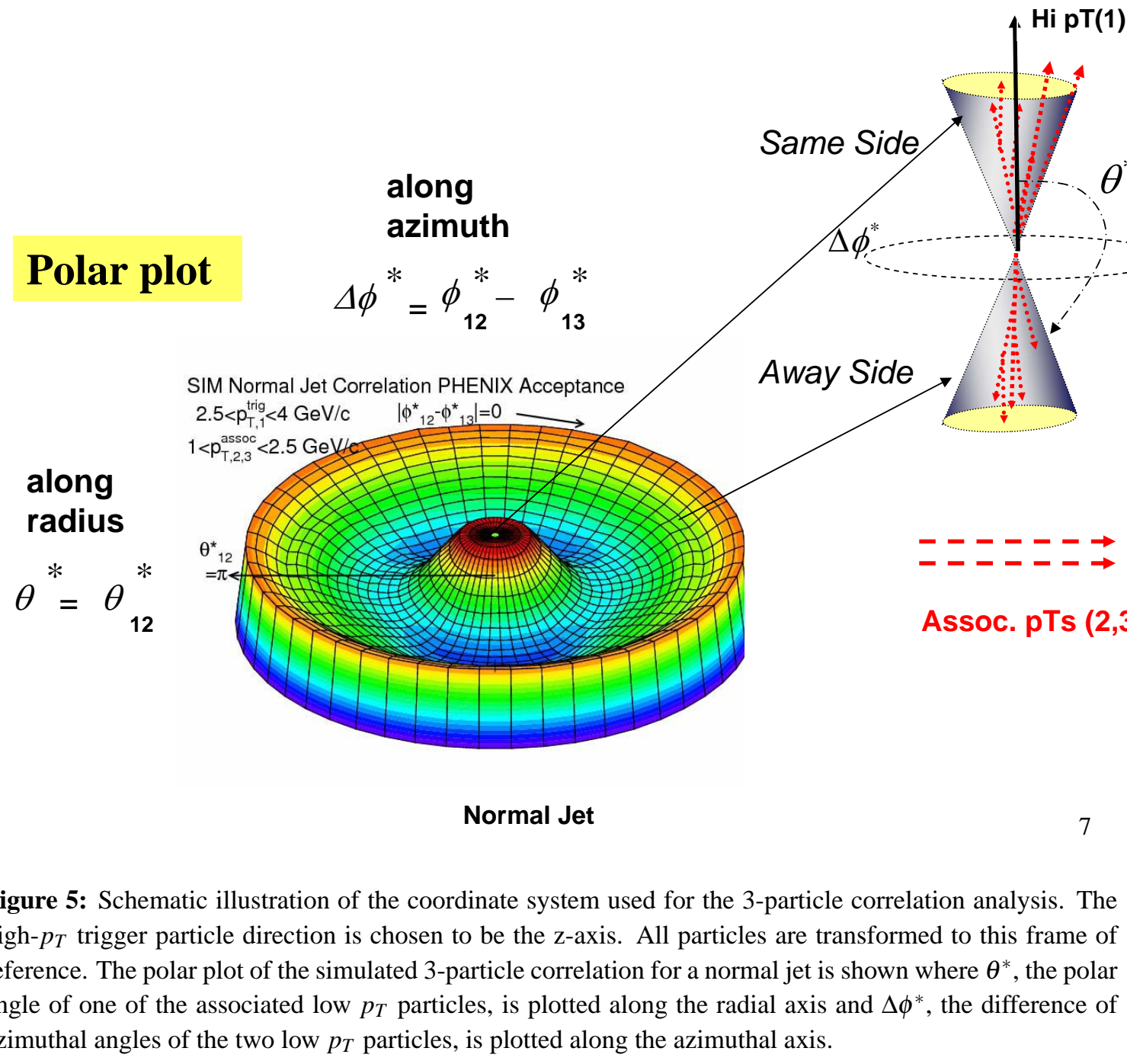

procedure[5]. Fig. 7 shows the efficiency of the ZYAM procedure in recovering the input jet topology.

The 3-particle jet correlations $C 3_{T J}$ obtained after removal of the flow effects have " $2+1$ " type jet correlations i.e. where two particles belong to the same jet and the third one does not. To remove " $2+1$ " correlations a 3-particle correlation, $C 3_{2 J}$, is constructed from two events where "real" triplets are made by taking two particles from one event and the third particle from the second event. The mixed triplet is (as before) made by taking each particle from a different event. The apportionment of two particle samplings between high pt-low pt pairs and low pt-low pt pairs reflect the natural proportion of such pairs in a standard two particle analysis. The final " $2+1$ " corrected triplet correlation $C 3_{J}$ is obtained by

$$
C 3_{J}=C 3_{T J} / C 3_{2 J}{ }^{R}
$$

where $R$ is the dilution of " $2+1$ " type correlations in the two-event correlation $C 3_{2 J}$. The dilution occurs because the 3-particle correlation constructed from two events not only breaks all true 3-particle correlations but also breaks a fraction of the 2-particle correlations thereby reducing 
High pT(1)

(a)

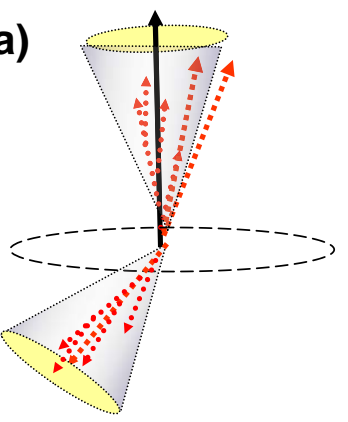

Deflected jet sim

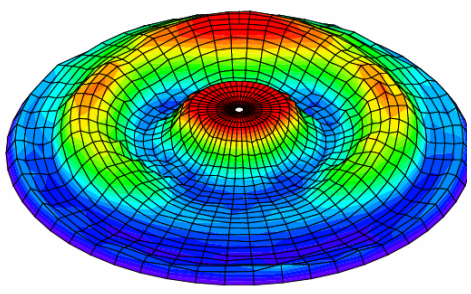

Deflected and Cone Jet Sims

(b)

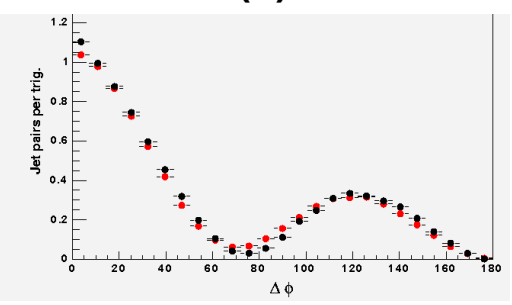

\section{2-particle Correlations matched}

High pT(1)

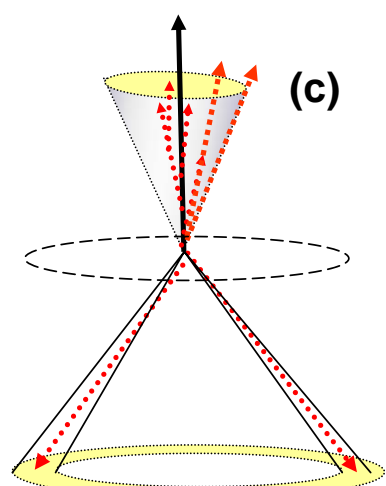

Mach Cone sim

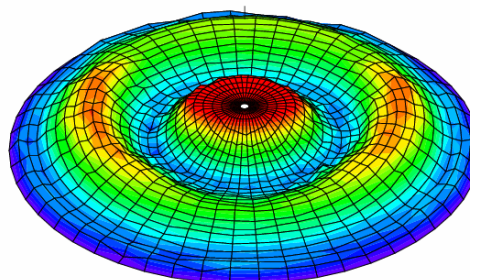

Figure 6: Schematic illustration of the correlation in the PHENIX acceptance for (a) deflected away-side jet and (c) a conical away-side jet. Note that the peaking of correlation strength in the $\Delta \phi^{*}=0$ region is characteristic of the deflected jet. Note also that the standard 2-particle azimuthal correlations (b) for the two cases are closely matched.

(a)

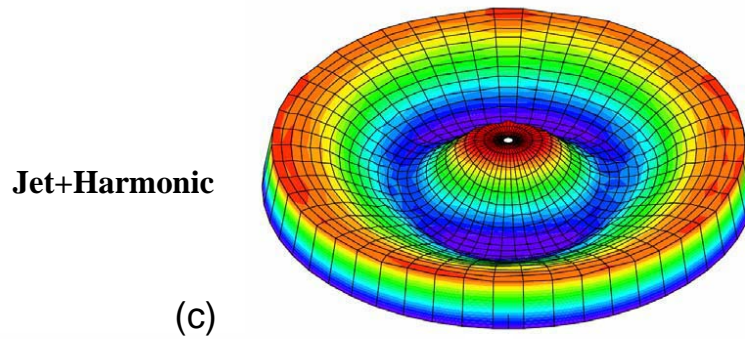

(b)

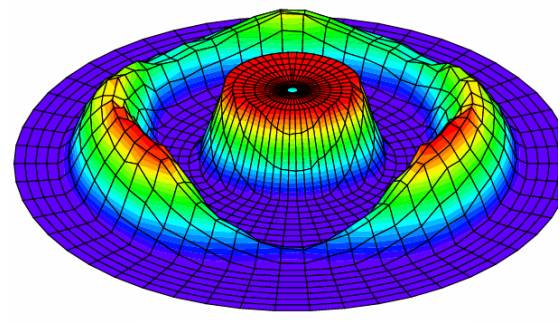

Harmonic removed

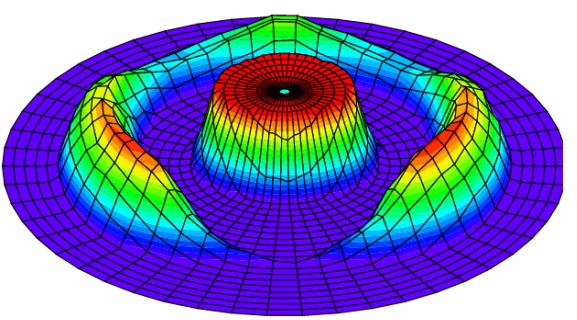

Input Jet

Figure 7: Demonstration of the ZYAM recovery of the input 3-particle jet correlation: (a) total (jet+harmonic) correlation function (b) with harmonic removed by ZYAM and (c) input jet function. 
the strength of the "2+1" correlations in relation to what is present in $C 3_{T J}$. The value of $R$ is determined by the ratio of low pt-low pt pairs to high pt-low pt pairs. Fig. 8a shows that for a case where true 3-particle correlations have been selectively removed, the removal of " $2+1$ " processes results in a flat correlation surface as expected. Fig. $8 \mathrm{~b}$ shows the faithful recovery of the true 3 -particle correlation after removal of the effects of harmonic and " $2+1$ " processes.

(a)

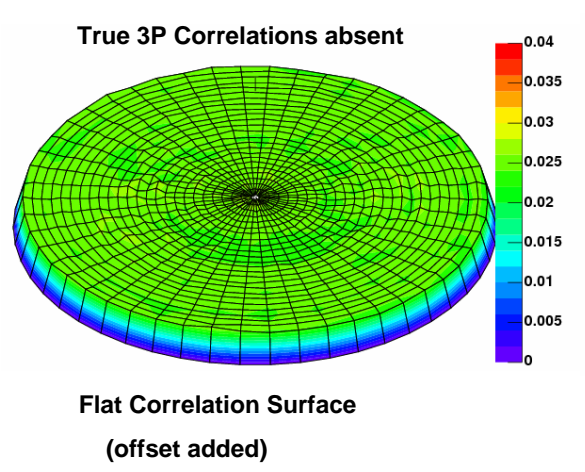

\section{(b)}

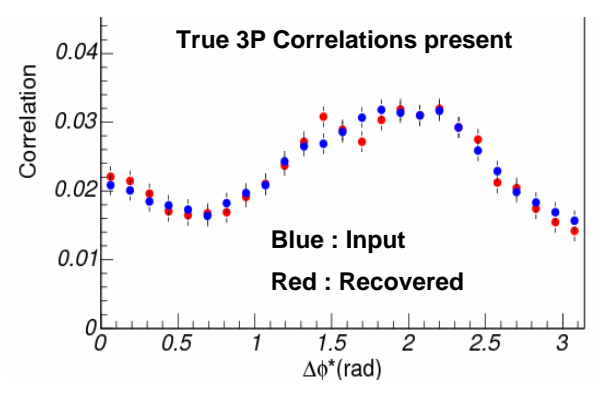

Figure 8: (a)Three particle correlation surface after removal of " $2+1$ " effects for a case where true 3-particle correlations have been selectively decorrelated. The surface is flat as expected showing the effectiveness of the " $2+1$ " removal method.(b)True 3-particle correlation recovered after removal of harmonic and " $2+1$ " contributions corresponds closely to the true input 3-particle correlation.

Fig. 9 shows the input true triplet yield and recovered true triplet yield showing the ability of the procedure to recover the input jet strength at the quantitative level.

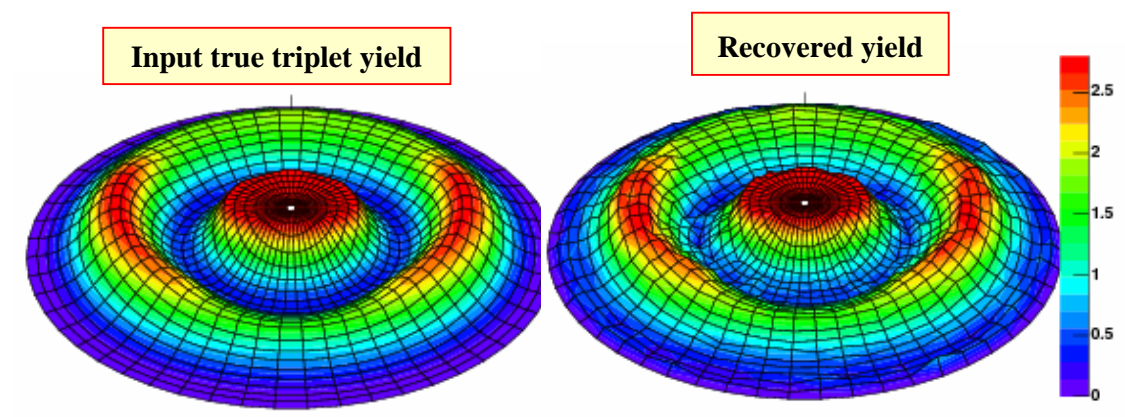

Figure 9: Input true triplet yield and recovered true triplet yield after removal of harmonic and $(2+1)$ contributions from the total correlation function

\subsection{Data Results:}

The full 3-particle correlation surface (i.e. inclusive of harmonic and " $2+1$ " effects) obtained from data for the $0-5 \%$ centrality selection in $\mathrm{Au}+\mathrm{Au}$ collisions is shown in Fig.10. It should be noted that because of the low flow signal at centrality $0-5 \%$, even without harmonic subtraction, 
the correlation surface is jet dominated. In this polar $\left(\theta^{*}, \Delta \phi^{*}\right)$ representation, one can clearly see the near-side jet correlations which are expected at the center of the correlation surface $\left(\theta^{*}=\right.$ $0)$. One can also see sizable correlations in $\Delta \phi^{*}$ for $\theta^{*} \sim 120^{\circ}$, albeit with acceptance losses especially in the region about $\left(\Delta \phi^{*} \sim 180^{0}\right)$. It is apparent that the away-side jet shows a shape which is significantly different from that expected from a normal jet where one expects enhanced correlations for $\theta^{*} \sim 180^{\circ}$.

\section{PHENIX Preliminary}

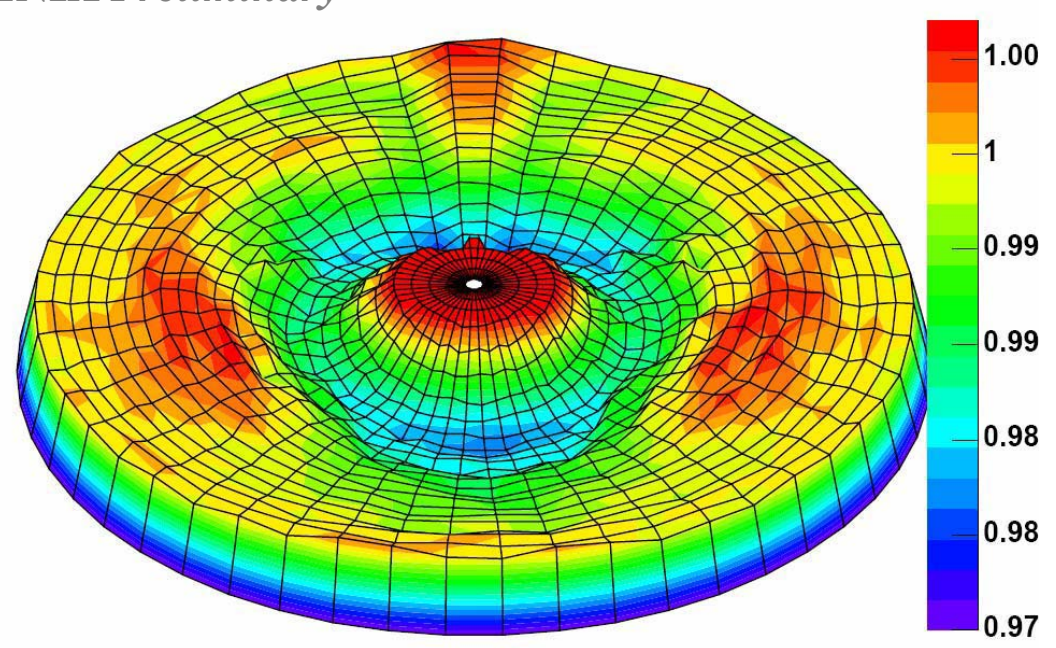

Total 3-particle Correlation $0-5 \%$ centrality

Figure 10: Full 3-particle correlation from the data for 0-5\% centrality selection in Au+Au collisions. Though harmonic correlations have not been removed they are small so that the correlations are jet dominated. The features seen are in stark contrast with what is expected for normal jets.

Fig.11 shows the resulting correlation surface for the centrality selection 10-20\% in $\mathrm{Au}+\mathrm{Au}$ collisions after removal of the harmonic contributions. The radial projection of the correlation at a specified value of $\Delta \phi^{*}$ (converted to triplet yield per trigger) shows a striking peak away from $180^{\circ}$. These results provide compelling evidence for strong modification of the away-side jet and provide important constraints on properties of the hot QCD matter such as the shear viscosity and speed of sound [7].

Fig. 12a and Fig. 12b [6]. show simulated true 3-particle correlation surfaces for deflected and cone scenarios respectively along with the azimuthal projections at the ridge in $\theta^{*}$ indicated by the ring. The jet to background multiplicity ratio has been adjusted to give 2-particle correlation strengths similar to that in the data. Also shown are the data for the corresponding azimuthal projection (as triplets per trigger) of the 3-particle correlation for the semi-central (10-20\%) $\mathrm{Au}+\mathrm{Au}$ collisions in the PHENIX acceptance after removal of effects due to flow and " $2+1$ " processes as detailed above. Here the data is clearly consistent with the Mach cone scenario but does not rule out the admixture of other topologies. 

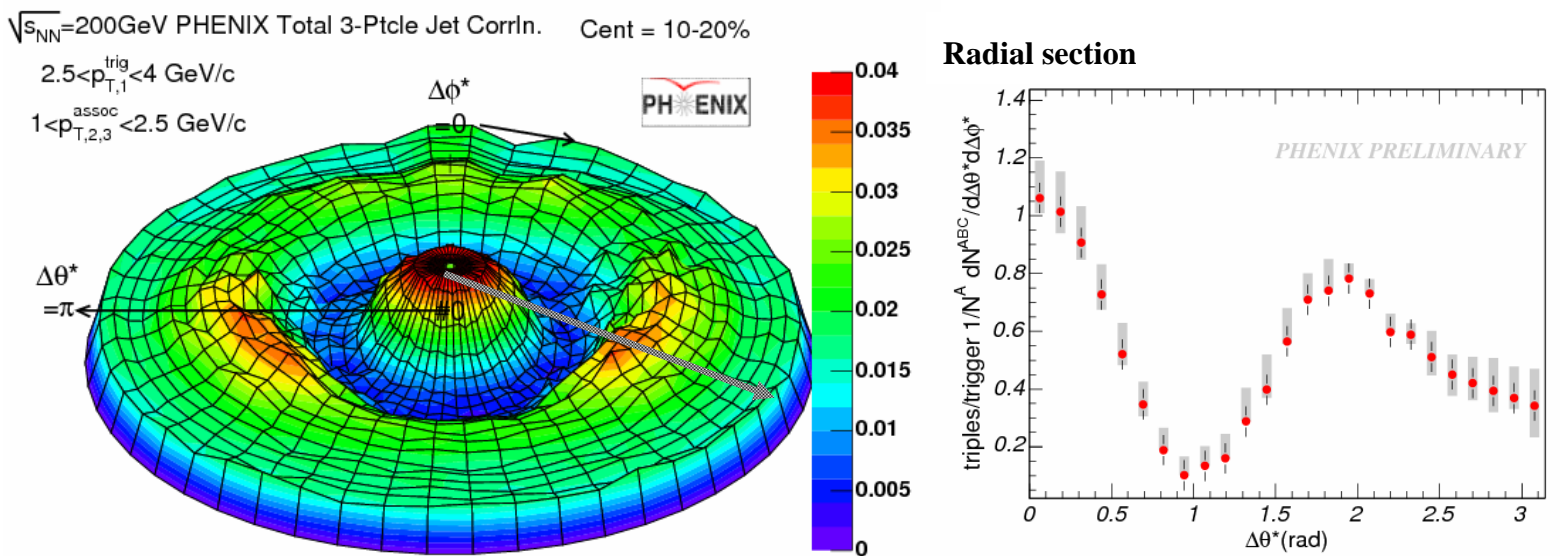

PHENIX Preliminary

\section{Total 3-particle Jet Correlation}

Figure 11: Flow subtracted total 3-particle correlation surface for charged hadrons detected in semi-central (10-20\%) Au+Au collisions within the PHENIX acceptance. The radial projection of the correlation shown as a triplet yield per trigger for the PHENIX acceptance at a specified value of $\Delta \phi^{*}$ clearly demonstrates the strong away side jet modification.

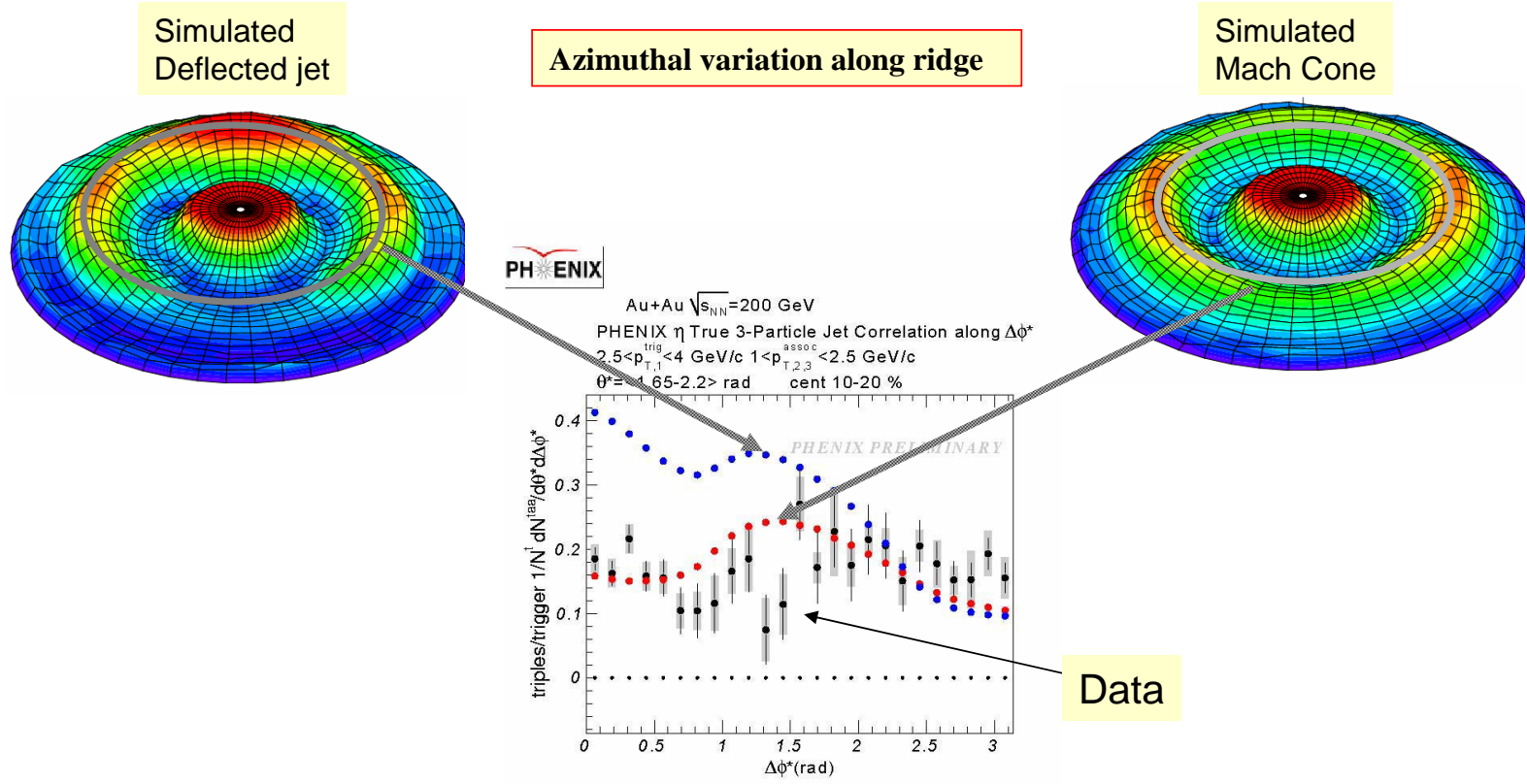

Figure 12: Figure shows simulated correlation surfaces for true 3-particle jet correlations for (a) deflected and (b) cone scenarios along with $\Delta \phi^{*}$ projection plots at the ridge in $\theta^{*}$ indicated by the rings. The corresponding projection plot for charged hadrons in semi-central (10-20\%) Au+Au collisions within the PHENIX acceptance is also shown as jet triplet yield per trigger. The data plot compares favorably with the expected shape for a Mach cone scenario. Note that the intensity scale of the polar plots is different from that in Figure 11 


\subsection{Looking ahead at LHC:}

Although the initial temperature of the matter formed at the LHC may be much higher, it is very likely that its subsequent trajectory in phase space will overlap the conditions seen at RHIC. In such a case, one may well see similar medium modification effects. A typical simulated LHC event is shown in Fig. 13 where the transverse energy $E_{T}$ is plotted for a large acceptance detector in $\eta-\phi$ space. After removal of the flow-associated soft background one can expect to see a number of outstanding peaks corresponding to high $\mathrm{pT}$ jets.

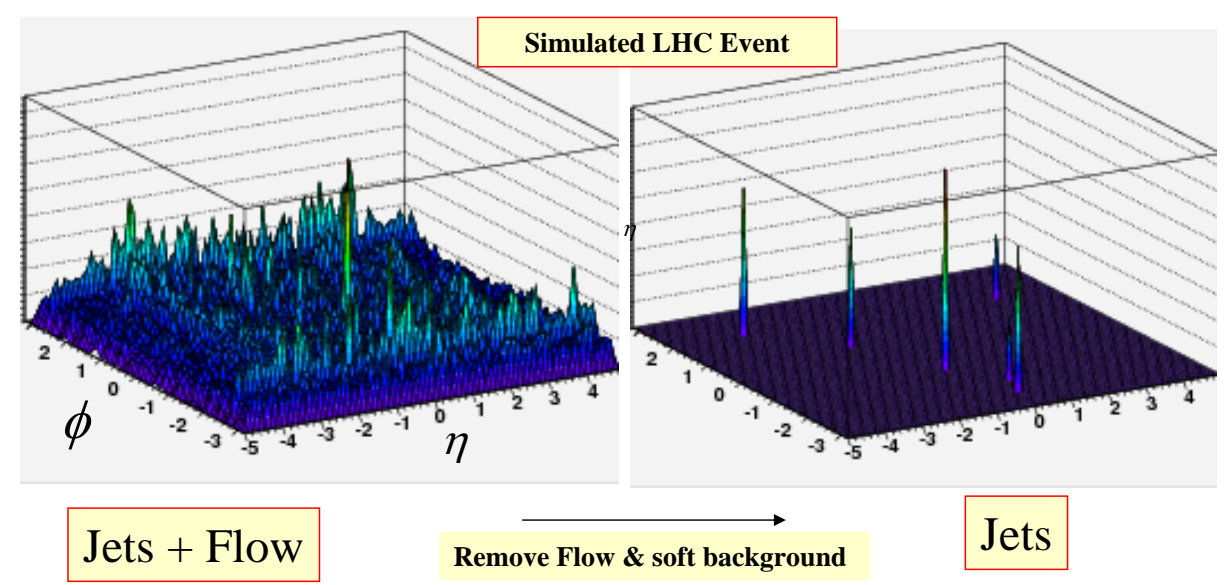

Figure 13: Simulated LHC event carrying contributions from both hard and soft processes (left) and after removal of soft contributions (right).

One can use these lead jet candidates from a large number of events to construct a 3-particle correlation of the type outlined above. Fig. 14 shows a typical topology to be expected of a Mach flow presence.

\subsection{Conclusions:}

The total 3-particle jet correlations obtained in $200 \mathrm{GeV} \mathrm{Au+Au}$, central case (0-5\%), though uncorrected for " $2+1$ " processes, displays an abnormal jet structure even without harmonic removal. The harmonic subtracted 3-particle correlation for 10-20\% centrality shows strong away side modification. The true 3-particle jet correlations obtained after removal of flow and "2 +1 " type jet contributions for the 10-20\% centrality is consistent with the presence of Mach Cone jets but do not rule out contributions from other topologies. The method may be a potential tool for jet study at the LHC.

\section{References}

[1] M. Gyulassy et al., Nucl. Phys. A750,30(2005); B. Muller (2004), nucl-th/0404015;E. Shuryak, Nucl. Phys. A750, 64 (2005);U. Heinz et al., Nucl. Phys. A702, 269 (2002)

[2] H. Stöcker, nucl-th/0406018; J. Casalderrey-Solana et al, hep-ph/0411315; B. Müller et al, Hep-ph/0503158; T. Renk et al, Hep-ph/0509036;

[3] Armesto,Salgado,Wiedemann hep-ph/0411341 


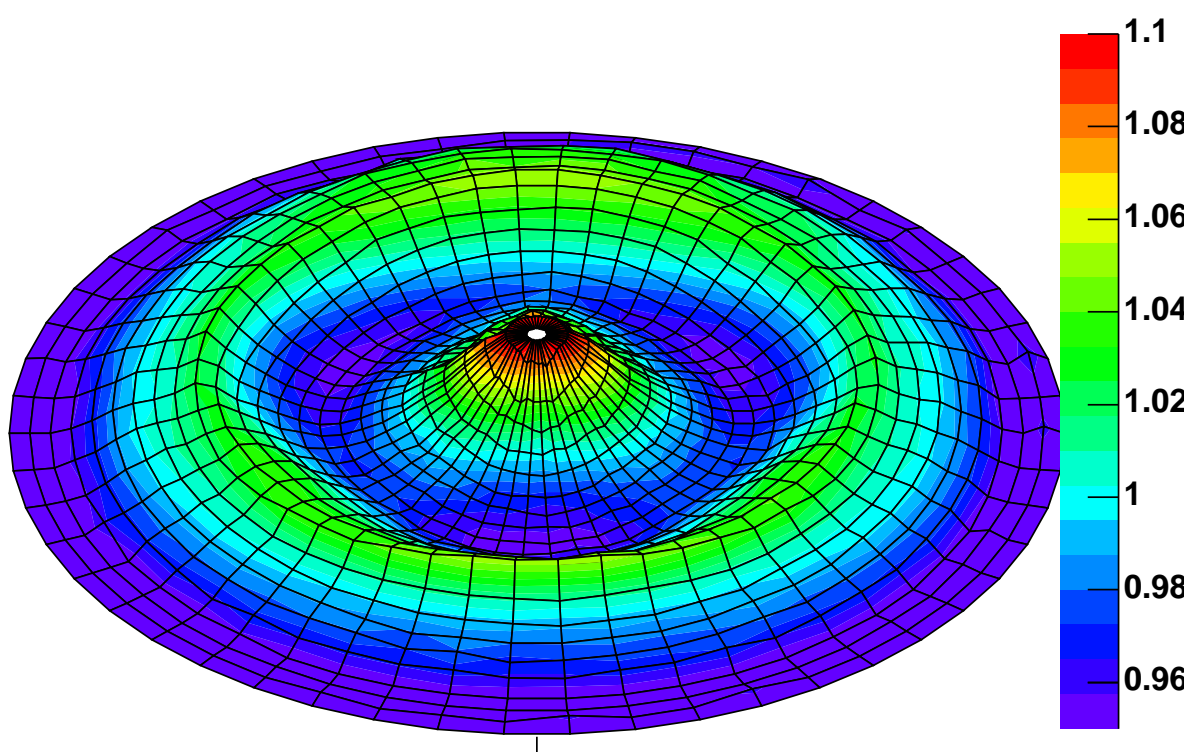

Figure 14: Expected correlation function shape for 3-particle correlations of in the leading jet frame for LHC events when the soft background has a Mach cone component.

[4] S. S. Adler et al. Phys. Rev. Lett. 97, 052301 (2006)

[5] N. Ajitanand, et al, Phys.Rev. C 72, 011902 (2005)

[6] C. Zhang for the PHENIX Collaboration, QM2006 Proceedings

[7] R. Lacey, nucl-ex/0608046; R. Lacey et al, nucl-ex/0609025 